\title{
Geometric Mechanics and the Dynamics of Asteroid Pairs
}

\author{
WANG-SANG KOON,${ }^{a}$ JERROLD E. MARSDEN,${ }^{a}$ SHANE D. ROSS,${ }^{a}$ \\ MARTIN LO, ${ }^{b}$ AND DANIEL J. SCHEERES ${ }^{c}$ \\ ${ }^{a}$ Control and Dynamical Systems, Caltech, Pasadena, California, USA \\ ${ }^{b}$ Navigation and Mission Design, Jet Propulsion Laboratory, \\ California Institute of Technology, Pasadena, California, USA
}

${ }^{c}$ Department of Aerospace Engineering, University of Michigan, Ann Arbor, Michigan, USA

\begin{abstract}
The purpose of this paper is to describe the general setting for the application of techniques from geometric mechanics and dynamical systems to the problem of asteroid pairs. The paper also gives some preliminary results on transport calculations and the associated problem of calculating binary asteroid escape rates. The dynamics of an asteroid pair, consisting of two irregularly shaped asteroids interacting through their gravitational potential is an example of a full-body problem or FBP in which two or more extended bodies interact. One of the interesting features of the binary asteroid problem is that there is coupling between their translational and rotational degrees of freedom. General FBPs have a wide range of other interesting aspects as well, including the 6-DOF guidance, control, and dynamics of vehicles, the dynamics of interacting or ionizing molecules, the evolution of small body, planetary, or stellar systems, and almost any other problem in which distributed bodies interact with each other or with an external field. This paper focuses on the specific case of asteroid pairs using techniques that are generally applicable to many other FBPs. This particular full two-body problem (F2BP) concerns the dynamical evolution of two rigid bodies mutually interacting via a gravitational field. Motivation comes from planetary science, where these interactions play a key role in the evolution of asteroid rotation states and binary asteroid systems. The techniques that are applied to this problem fall into two main categories. The first is the use of geometric mechanics to obtain a description of the reduced phase space, which opens the door to a number of powerful techniques, such as the energy-momentum method for determining the stability of equilibria and the use of variational integrators for greater accuracy in simulation. Second, techniques from computational dynamic systems are used to determine phase space structures that are important for transport phenomena and dynamic evolution.
\end{abstract}

KEYWORDS: geometric mechanics; dynamics; asteroid pairs; full-body problem

Address for correspondence: Shane Ross, Control and Dynamical Systems 107-81, Caltech, Pasadena, CA 91125, USA.

shane@cds.caltech.edu

Ann. N.Y. Acad. Sci. 1017: 11-38 (2004). (C2004 New York Academy of Sciences. doi: 10.1196/annals.1311.002 


\section{INTRODUCTION}

Full-body problems (FBPs) are concerned with the dynamical interaction between two or more distributed bodies. This fascinating class of problems has many interesting open questions and touches on numerous important issues in science and engineering. Examples include binary asteroids, the evolution and dynamics of the Earth-Moon system, the dynamics and control of a high-performance aircraft, reaction and ionization of molecules, interactions and collisions between galaxies, stability and control of underwater vehicles, rendezvous and docking of space vehicles, and fine pointing control of a space-based telescope. Geometric mechanics and dynamical systems theory, together with appropriate computational and geometric control techniques, provide a unified approach to the analysis and simulation of these problems.

There are many examples in which geometric mechanics methods have been used for this general class of problems. Two examples are the work concerning the use of reduction and stability methods for rigid bodies with flexible attachments ${ }^{1}$ and also the work on the use of the energy-momentum method for tethered satellites. ${ }^{2}$

\section{The Binary Asteroid Problem}

In this paper we focus on the dynamics of binary asteroids in the context of F2BPs. This problem concerns the dynamics of two spatially extended bodies that interact via their mutual gravitational fields. An example of a motivating goal is the accurate estimation of ejection, collision, and transport rates, accounting fully for coupling between the rotational and translational states of the bodies. We put this problem into the context of systematic approaches to determine the stability of relative equilibria, as well as the computation of phase space structures, such as periodic orbits, quasi-periodic orbits, and the division of phase space into regions of regular and chaotic motion. This context allows one to bring to bear powerful computational transport techniques, such as set-oriented methods and lobe dynamics. As we review below, there is already quite a bit known about relative equilibria in the binary asteroid problem; we refer to Reference 3 for additional information. However, rather little has been done on the energy-momentum method (and its converse) for relative equilibria, as well as the problem of merging geometric mechanics and dynamical systems calculations, including phase space structure. Our goal is to take some first steps in this direction, but much work remains to be done.

The binary asteroid problem is of considerable astrodynamic interest. For instance, the methods are directly relevant to asteroid rotational evolution, variation of planetary obliquities, and the long-term dynamics of Kuiper and asteroid belt binaries, including an analysis of the Pluto-Charon system.

\section{A Little Biased History}

Special cases of the F2BP have been analyzed extensively in the literature, with most applications focusing on the interaction of a small, distended body with a much larger body, for example the evolution of irregularly shaped planetary moons or the dynamics and control of gravity gradient spacecraft. Even for this simplified version of the F2BP it is well-known that chaotic motion exists and can play an essential role 
in the life of a tidally evolving body (see, for instance, Refs. 4 and 5). Studies from the astrodynamics community have discovered many fundamental symmetries that exist in these ideal problems, ${ }^{6-9}$ which have in turn been used to develop novel methods for the control of these systems. ${ }^{10-12}$ The other extreme case of the F2BP has also been investigated, namely the motion of a massless particle in the field of a strongly non-spherical gravity field, with main application to the motion of spacecraft and ejecta relative to an asteroid, comet or moon. ${ }^{13-19}$

An early paper on the FBP was by Duboshin, ${ }^{20}$ although his subsequent studies focused on the force laws necessary for certain solutions and symmetries to exist. Maciejewski $^{21}$ gives a modern statement of the F2BP, along with a preliminary discussion of relative equilibria possible for these systems, but with no investigation of the dynamic evolution and stability of such problems. More recently, sharp conditions for the Hill and Lagrange stability of the F2BP have been obtained ${ }^{22}$ and numerical and analytical investigations of rotational and translational coupling in these problems have been studied. ${ }^{23-25}$ These most recent works serve as one starting point for the current paper.

\section{TOOLS FOR THE FBP}

The F2BP is a magnificently symmetric problem with an overall symmetry group $S E(3)$, the group of Euclidean motions in three-space, together with any symmetries of the bodies themselves. Thus, it is ideal for using the tools of geometric mechanics for systems with symmetry, variational integrators, and transport and dynamical systems theory. In this paper we take some small initial steps in this direction for simplified, but still non-trivial, versions of the F2BP. It is our hope that this effort will help merge work from a number of groups into a single coherent theory. Some of the salient features of these methods are as follows.

1. Geometric mechanics and reductions of the F2BP: As hinted at already and as explained further elsewhere ${ }^{26-28}$ geometric mechanics has had enormous successes in many areas of mechanics. Previous investigations in the FBP, however, have been accomplished in a fragmentary way and have missed using, for example, the powerful energy-momentum method and its converse for relative equilibria, as well as the use of geometric phases. For example, the converse of the energy-momentum method ${ }^{29,30}$ allows one to study the destabilizing effect of dissipation and body deformations for relative equilibria that are gyroscopically stabilized on the linear level (that is, one has a saddle point in the augmented energy, but the system eigenvalues are on the imaginary axis, so one cannot easily infer stability or instability in the nonlinear system).

2. Variational integrators for the F2BP: A novel computational technique that is ripe for use on the FBP is that of asynchronous variational integrators (AVIs). These are numerical simulation methods that allow different time steps at different spatial points and yet have all the advantages of the usual symplectic integrators used in dynamical astronomy (see, for instance, Refs. 31 and 32 and citations therein). For example, in many FBPs there are large differences in time scales for the dynamics of rotational and translational motions. AVIs, which allow for such adaptation, greatly improve computation efficiency, as has been 
demonstrated in related problems, ${ }^{33,34}$ and hence permit new problems to be directly simulated for longer times and to higher accuracy than previously achieved.

3. Phase space structure and transport calculations: A third key tool that can be brought to bear on this problem concern advances in the computation of transport rates using transition state theory borrowed from chemistry and lobe dynamics from fluid dynamics, where recent computation advances have enabled significant capabilities using these approaches. ${ }^{35}$ This may be combined with set oriented methods involving concepts from graph theory, such as the notion of graph partitions, congestion, almost invariant sets, and the Peron-Frobenius operator. ${ }^{36}$ Drawing on recent advances in computing phase space objects, such as periodic orbits and invariant tori for astrodynamic problems, it would be interesting to investigate the link between periodic orbits, resonances, and restrictions (pinch points) in the phase space of the F2BP, structures important for the computation of transport, such as ejection and collision rates.

There are a number of fundamental questions in planetary science that can be addressed by the techniques that we outline, including: comet nucleus rotational and translational evolution due to outgassing; long-term simulation and evaluations of the Yarkovsky effect on the rotational and translational motion of asteroids; tidal evolution of asteroid and Kuiper belt binaries, including dissipation and external forces; dynamic evolution of galaxies that undergo a close approach.

\section{THE F2BP AND ASTEROID BINARIES}

Although problems involving rotational and translational coupling populate many areas of science and engineering, for tractability in this paper we draw on examples that arise in the field of planetary science and dynamical astronomy, because there are many FBPs in this field whose complete understanding is still lacking and whose simulation depends on gross physical approximations to certain aspects of their motion. Examples include the evolution of the obliquities of the terrestrial planets, ${ }^{5,37}$ the rotational dynamics of Europa, ${ }^{38}$ the effect of the Yarkovsky force on translational and rotational evolution of asteroids, ${ }^{39-42}$ and the evolution of comet nucleus rotation and translation due to outgassing, ${ }^{43,44}$ to name a few. In each of these cases interactions between rotation and translation are important, but detailed simulation and evaluation of these couplings over long time spans is beyond current capability. More importantly, the specialized force environments and physical effects unique to each of these systems has made it difficult to formulate a general, unifying approach to the analysis and simulation of these problems.

Recent studies of the dynamics and evolution of binary asteroids, provides a class of problems that can serve as fundamental, or canonical, models that are key stepping stones to a unified approach to FBPs. This class of problems are of interest in their own right, since recent analysis indicates that up to $20 \%$ of near-Earth asteroids are binaries, ${ }^{45}$ along with the current boom of binary discoveries in the main asteroid belt and the Kuiper belt ${ }^{46,47}$ (see FIGURE 1). The analysis and modeling of binary asteroids (or similarly binary Kuiper belt objects) does not allow the usual assumptions of small gravity coefficients, near equilibrium conditions, or long-term stability 

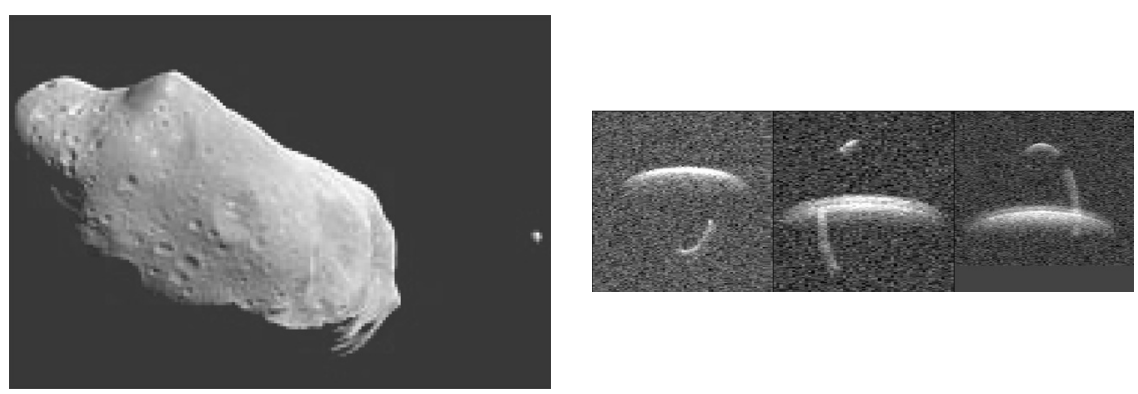

FIGURE 1. Dactyl in orbit about Ida, discovered in 1994 during the Galileo mission, ${ }^{48}$ and 1999 KW4 radar images of its companion, discovered in 2001.49

of motion made in classical approaches to FBPs. Rather, to properly understand the dynamics and evolution of these systems requires a generalized approach to the problem that incorporates these difficulties into its nominal problem statement.

\section{Recent Advances}

The general F2BP has been studied under many different approximations, but its general statement has only relatively recently been posed, ${ }^{21}$ followed by advances in understanding its Hill and Lagrange stability, ${ }^{22}$ and observations on the role of these interactions on the evolution of small body rotation states. ${ }^{23,25,50}$ The coupled motion in this problem can lead to profound dynamics, illustrated in FIGURE 2, ${ }^{25}$ showing the evolving orbit radius and rotation period of a sphere and triaxial ellipsoid of equal mass interacting through gravity alone. The total energy of this system is slightly negative, meaning that the two bodies can never mutually escape (that is, they are Hill stable). The system is also stable against impact (Lagrange stable), and so will evolve ad infinitum unless some small external perturbation (such as from the Sun) boosts its energy sufficiently to allow for escape, which would also leave the ellipsoid with an extremely slow rotation rate. The phase space of this apparently chaotic system can be tightly constrained using integrals of motion, and separated regions of phase space can be identified as a function of resonances between the rigid-body rotation of the system and orbital rotation rates. FIGURE $2 \mathrm{C}^{25}$ shows an example of the allowable phase space of this system, with additional restrictions on motion in the phase space, identified as "pinch points" in the figure, that arise from these resonances. We conjecture that such pinch points can be understood via the reduction process from geometric mechanics and that these will be important in understanding transport and ejection rates.

\section{Models In Use}

The equations of motion for the F2BP model may be explicitly found and are discussed in a number of references. ${ }^{21,22} \mathrm{~A}$ widely used model can be derived when one of the bodies is a massive sphere. The rotational dynamics of the sphere decouples from the system, which still has coupling of rotational and translational dynamics 
between the non-spherical body and the relative orbit between the two. The normalized and symmetry reduced equations are

$$
\begin{gathered}
\mathbf{r}^{\prime \prime}+2 \omega \times \mathbf{r}^{\prime}+\omega^{\prime} \times \mathbf{r}+\omega \times(\omega \times \mathbf{r})=\frac{\partial \mathcal{U}}{\partial \mathbf{r}} \\
I \cdot \omega^{\prime}+\omega \times I \cdot \omega=-\mu \mathbf{r} \times \frac{\partial \mathcal{U}}{\partial \mathbf{r}},
\end{gathered}
$$

where

$$
\begin{aligned}
\mathcal{K} & =\frac{1}{\mu} \mathbf{A} \cdot I \cdot \omega+\mathbf{A} \cdot \mathbf{r} \times\left(\mathbf{r}^{\prime}+\omega \times \mathbf{r}\right) \\
\mathcal{E} & =\frac{1}{2}\left(\mathbf{r}^{\prime}+\omega \times \mathbf{r}\right) \cdot\left(\mathbf{r}^{\prime}+\omega \times \mathbf{r}\right)+\frac{1}{2 \mu} \omega \cdot I \cdot \omega-\mathcal{U}
\end{aligned}
$$

and where $\omega$ is the rotational velocity vector in the body-fixed frame, $\mathbf{r}$ is the relative position vector in the body-fixed frame, $\mathbf{A}$ is the attitude tensor of the non-spherical body, $I$ is the specific inertia tensor of the non-spherical body, $\mathcal{U}$ is the gravitational force potential of the non-spherical body, and $\mathcal{K}$ and $\mathcal{E}$ are the angular momentum and energy integrals, respectively, of these equations.
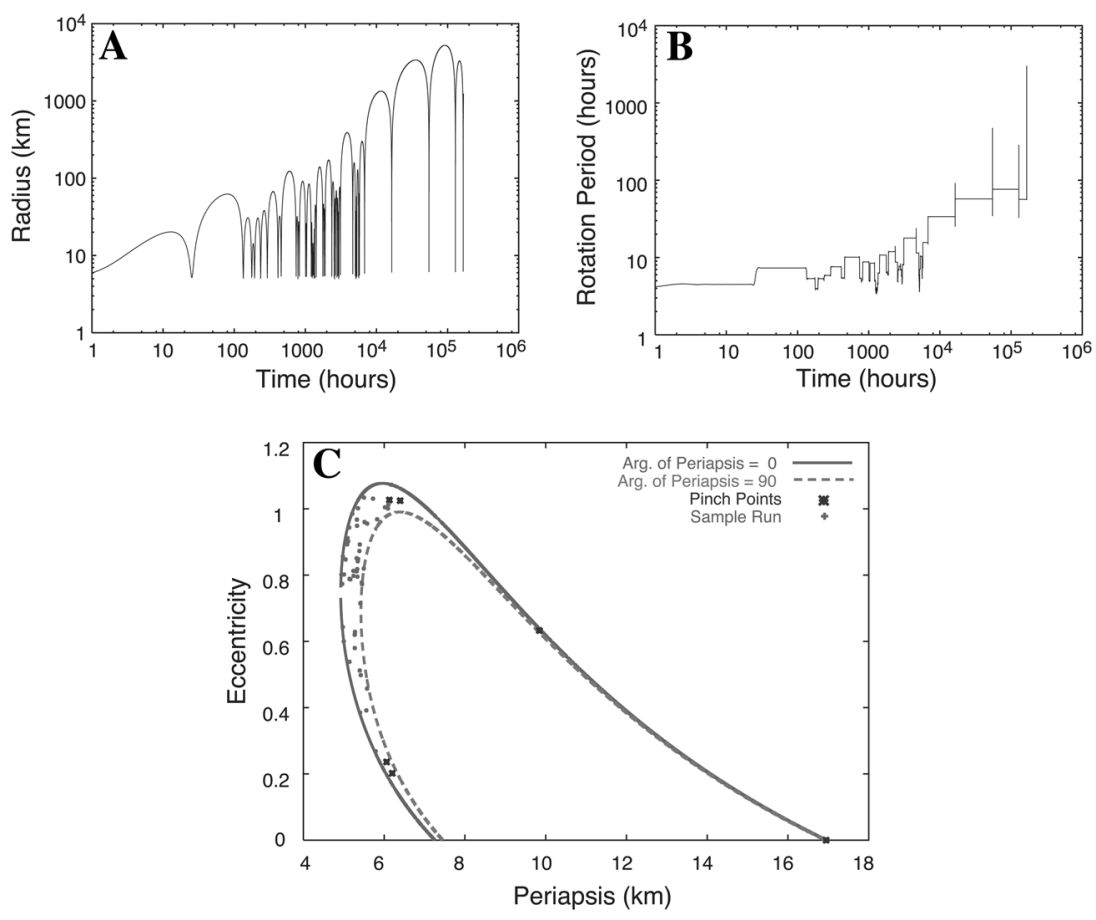

FIGURE 2. Time history of the orbit radius (A) and rotation period (B) for a gravitationally interacting sphere and tri-axial ellipsoid of equal mass. Poincaré map (C) showing constraints on the eccentricity of the evolving orbit, including pinch points that isolate regions of phase space. 
The free parameters of the system are the mass distribution of the non-spherical body (normalized by its largest dimension) and the mass fraction between the two bodies, $\mu=M_{1} /\left(M_{1}+M_{2}\right)$ (the same parameter found in the restricted three-body problem), where $M_{1}$ is the mass of the "sphere" and $M_{2}$ is the mass of the distributed body. The case when $\mu \rightarrow 1$ corresponds to the motion of a massless distributed body about a point-mass, with an application to a satellite in orbit about a planet. It is important to note that the angular momentum and energy integrals still apply to this problem. The case when $\mu \rightarrow 0$ corresponds to the motion of a material point in the gravity field of the distributed body, with the main application to particle dynamics about an asteroid. In this case, we see that the energy and angular momentum integrals are dominated by the rotational dynamics of the distributed body, and that the contribution of the motion of the spherical body decouples from these integrals.

In this paper we use Equation (1), often with additional simplifications. These simplifications are made to allow for an abbreviated discussion of results and will be relaxed in future papers. The main simplification made is the restricted, uniformly rotating approximation that occurs when $\mu \rightarrow 0$ and the distributed body (which now has all the mass of the system) is in principal axis rotation about its maximum moment of inertia (assumed to lie along the $z$-axis). When needed, we refer to this system as the restricted full two-body problem (RF2BP), and we note its similarity to the celebrated restricted three-body problem, including the existence of a Jacobi integral. The RF2BP, although highly idealistic, is important for understanding the general and qualitative properties of motion about distended bodies in uniform rotation $^{51}$ and approximately models the motions of some binary asteroids.

Carrying out such detailed calculations over the time scales of interest for the F2BP is challenging and requires methods designed to conserve the symmetries inherent in the problem and to handle the multiple time scales in FBPs. Additionally, the existence and prediction of the restricted regions of phase space far from an equilibrium point is an example of a complex, poorly understood dynamic phenomenon. The techniques discussed below take some first steps to address this deficiency. The F2BP is ideally suited for the application of geometric mechanics with symmetry, variational integrators, and transport and dynamic systems theory. Due to the generality of its statement, the F2BP can be extended to cover other systems of interest in planetary science, dynamical astronomy, astrodynamics, and chemical dynamics, briefly discussed later in the paper.

\section{Geometric Mechanics and the F2BP}

We ultimately want to carry out an analysis of the F2BP by first looking at the symmetry of the problem. The dynamics of the F2BP are invariant under application of orientation-preserving rotations and translations of three-dimensional Euclidean space; that is, under the application of the symmetry group $S E(3)$. Thus, in the terminology of geometric mechanics, we say that the F2BP carries the symmetry of invariance with respect to $S E(3)$. A well-known property of mechanical systems like the F2BP is that whenever there is a symmetry, there are corresponding conserved quantities.

As the symmetry group becomes larger, we expect that one recovers all the special cases studied in the literature. For example, if one body is an irregular body and the other is cigar-like, the symmetry group is $S E(3) \times S^{1}$. Relative equilibria and 
their stability can be studied using the powerful energy-momentum method. ${ }^{28,30,52}$ Relative equilibria are key ingredients in identifying and characterizing the possible final states that an evolving binary asteroid can reach and they play a similar role to libration points in the three-body problem. Moreover, a systematic geometric mechanical approach will enable the use of modern numerical algorithms, such as variational integrators, in the accurate computations of relevant long term statistics and transport. $33,34,53$

\section{Symmetry Reduction}

The use of geometric and dynamic techniques to study Lagrangian or Hamiltonian mechanics has been enormously successful in a wide variety of engineering and astrodynamic problems, such as the use of the energy-momentum method for stability of satellites with internal rotor controls, ${ }^{30}$ and in heteroclinic and resonance structures in the three-body problem. ${ }^{54}$ Geometric mechanics starts with the usual formulation of Lagrangian mechanics using variational principles, and Hamiltonian mechanics using Poisson and symplectic geometry. Lagrangian reduction by a symmetry group corresponds to finding reduced variational principles, whereas reduction on the Hamiltonian side corresponds to constructing appropriate reduced Poisson and symplectic structures.

The reduction of a system with configuration manifold $Q$ and Lie symmetry group $G$ occurs at two levels, the first of which corresponds to identifying solutions that are related by a symmetry group motion, and this corresponds to deriving equations of motion on the quotient space $T Q / G$ (for Lagrangian reduction), and on $T^{*} Q / G$, (for Hamiltonian reduction). Symmetry gives an associated Noether theorem, namely conservation of a momentum map, so that the dynamics are constrained to a momentum surface in the absence of external forcing and dissipation. When appropriate, imposing this constraint explicitly in the equations of motion, yields a further reduction in the dimensionality of the reduced equations, which corresponds to (nonabelian) Routh and symplectic reduction.

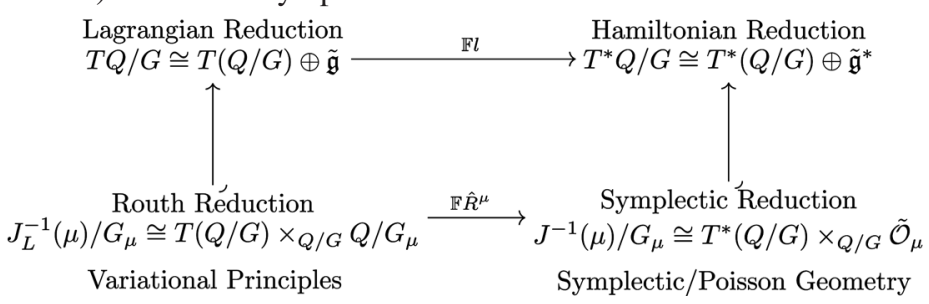

On the Lagrangian side, general theorems give the structure of the quotient space to be

$$
T Q / G \cong T(Q / G) \oplus \tilde{g},
$$

where $g$ is the Lie algebra of $G$ and where $\tilde{g}$ is an associated bundle over shape space $Q / G$, as shown in Reference 55 . There is a similar structure theorem on the Hamiltonian side. When the momentum constraints are imposed, on the Hamiltonian side one gets well known cotangent bundle reduction theorems, ${ }^{28}$ whereas on the Lagrangian side one gets a far reaching, and surprisingly recent, generalization of the classical Routh procedure. ${ }^{56}$ The general scheme of the reduction procedure is 
shown in the above diagram. For the F2BP, the choice of $Q$ and $G$ are easily made and are given in the next section.

\section{Reduction for the F2BP}

For the F2BP, the configuration space is $Q=S E(3) \times S E(3)$. Denote material points in a reference configuration by $X_{i}$, and the points in the current configuration by $x_{i}$. Given $\left(\left(A_{1}, r_{1}\right),\left(A_{2}, r_{2}\right)\right) \in S E(3) \times S E(3)$, points in the reference and current configurations are related by $x_{i}=A_{i} X_{i}+r_{i}, i=1,2$. Using the body angular velocity notation defined previously, the Lagrangian has the standard form of kinetic minus potential energy:

$$
\begin{aligned}
& L\left(A_{1}, r_{1}, A_{2}, r_{2}\right)=\frac{1}{2} \int_{\beta_{1}}\left\|\dot{x}_{1}\right\|^{2} d \mu_{1}\left(X_{1}\right)+\frac{1}{2} \int_{B_{2}}\left\|\dot{x}_{2}\right\|^{2} d \mu_{2}\left(X_{2}\right) \\
& +\int_{B_{1}} \int_{B_{2}} \frac{G d \mu_{1}\left(X_{1}\right) d \mu_{2}\left(X_{2}\right)}{\left\|x_{1}-x_{2}\right\|} \\
& =\frac{m_{1}}{2}\left\|\dot{r}_{1}\right\|^{2}+\frac{1}{2}\left\langle\Omega_{1}, I_{1} \Omega_{1}\right\rangle+\frac{m_{2}}{2}\left\|\dot{r}_{2}\right\|^{2}+\frac{1}{2}\left\langle\Omega_{2}, I_{2} \Omega_{2}\right\rangle \\
& +\int_{B_{1}} \int_{B_{2}} \frac{G d \mu_{1}\left(X_{1}\right) d \mu_{2}\left(X_{2}\right)}{\left\|A_{1} X_{1}-A_{2} X_{2}+r_{1}-r_{2}\right\|} \text {. }
\end{aligned}
$$

\section{Reducing by Overall Translations and Rotations}

The preceding Lagrangian has symmetry group $S E(3)$ using the diagonal left action on $Q$ :

$$
(A, r) \cdot\left(A_{1}, r_{1}, A_{2}, r_{2}\right)=\left(A A_{1}, A r_{1}+r, A A_{2}, A r_{2}+r\right) .
$$

The momentum map corresponding to this symmetry is the total linear momentum and the total angular momentum. As in References 21, 57, and 58, the projection from the configuration space to the shape space $\pi: Q \rightarrow Q / G$, is obtained by transforming to the body frame of the second rigid body. The reduction is carried out in stages, by first reducing by translations, $\mathbb{R}^{3}$, followed by reducing by rotations, $S O(3)$. Results from general reduction by stages $55,59,60$ shows that this is equivalent to directly reducing by $S E(3)$ in a single step. This is achieved by applying the inverse of $\left(A_{2}, r_{2}\right)$, which is given by $\left(A_{2}^{T},-A_{2}^{T} r_{2}\right) \in S E(3)$,

$$
\begin{aligned}
\left(A_{2}^{T},-A_{2}^{T} r_{2}\right) \cdot\left(A_{1}, r_{1}, A_{2}, r_{2}\right) & =\left(A_{2}^{T} A_{1}, A_{2}^{T} r_{1}-A_{2}^{T} r_{2}, A_{2}^{T} A_{2}, A_{2}^{T} r_{2}-A_{2}^{T} r_{2}\right) \\
& =\left(A_{2}^{T} A_{1}, A_{2}^{T}\left(r_{1}-r_{2}\right), e, 0\right) .
\end{aligned}
$$

Shape space $Q / G$ is isomorphic to one copy of $S E(3)$, being coordinatized by the relative attitude $A_{2}^{T} A_{1}=A^{T}$ and relative position $A_{2}^{T}\left(r_{1}-r_{2}\right)=R$. The equations of motion in $T(Q / G)$ (respectively, $T^{*}(Q / G)$ ) involve $A, R$, and their velocities (respectively), conjugate momenta $\Gamma, P$ ), which correspond to total angular and linear momenta in the body fixed frame of the second rigid body. These are coupled to equations in $\mathfrak{G e}(3)^{*}$, which may be identified with equations for the body angular and linear momenta of the second rigid body, $\Gamma_{2}, P_{2}$.

Elsewhere, ${ }^{21,57,58}$ the equations for $\Gamma$ and $\Gamma_{2}$ are rewritten in terms of $\Gamma_{1}, \Gamma_{2}$ and the linear momentum of the second rigid body is ignored. A reconstruction-like 
equation (that is, recovering the full attitude of both bodies) is added at the very end. One thing missing from the literature is the systematic use of shape space to study geometric phases that are important for rotational and translational drifts. ${ }^{59,61}$ The general context of geometric mechanics and the specific setting of the present paper should enable one to fill in this interesting gap. The coordinatization of the reduced space by transforming to the reference frame of the second rigid body corresponds to a particular choice of connection on the principal bundle $Q \rightarrow Q / G$. Different choices of connection will affect how we identify $T Q / G$ with $T(Q / G) \oplus \tilde{g}$.

The reduced Lagrangian is obtained by rewriting the Lagrangian in terms of the reduced variables:

$$
A=A_{2}^{T} A_{1}, \quad R=A_{2}^{T}\left(r_{1}-r_{2}\right), \quad \hat{\Omega}=A_{2}^{T} \dot{A}_{1}, \quad V=A_{2}^{T}\left(\dot{r}_{1}-\dot{r}_{2}\right),
$$

which are coordinates on $T(Q / G)$, as well as $\hat{\Omega}_{2}=A_{2}^{T} \dot{A_{2}}, V_{2}=A_{2}^{T} \dot{r}_{2}$, which are coordinates on $\mathfrak{S e}(3)$. As with the Euler-Poincaré theory, ${ }^{27}$ Hamilton's variational principle on $T(S E(3) \times S E(3))$ is equivalent to the reduced variational principle,

$$
\delta \int_{a}^{b} l\left(A, R, \hat{\Omega}, V, \hat{\Omega}_{2}, V_{2}\right) d t=0
$$

on $\mathbb{R}^{18}$ where the variations are of the form,

$$
\begin{gathered}
\delta A=-\hat{\Sigma}_{2} A+\hat{\Sigma}, \quad \delta R=-\hat{\Sigma}_{2} R+S, \quad \delta \hat{\Omega}=\dot{\hat{\Sigma}}-\hat{\Sigma}_{2} \hat{\Omega}+\hat{\Omega}_{2} \hat{\Sigma}, \\
\delta V=\dot{S}-\hat{\Sigma}_{2} V+\hat{\Omega}_{2} S, \quad \delta \Omega_{2}=\dot{\Sigma}_{2}+\Omega_{2} \times \Sigma_{2}, \quad \delta V_{2}=\dot{S}-\hat{\Sigma}_{2} V_{2}+\hat{\Omega}_{2} S_{2},
\end{gathered}
$$

and $S, S_{2}, \Sigma$, and $\Sigma_{2}$ are variations that vanish at the boundary. The symplectic form can be obtained from the variational principle by considering variations that do not vanish at the boundary. The boundary terms that arise from integration by parts can be interpreted as a Lagrange one-form, and taking the exterior derivative of these yield the symplectic two-form.

Already, the above approach is more systematic and complete than that given in the literature. Although simple, the variational structure just given is, in fact, new. In future studies, we will complete this task and deal with all of the systematic cases for the F2BP and to tie in this theory with the stability theory of relative equilibria using the powerful energy-momentum method.

\section{The Energy-Momentum Method}

This is a powerful generalization of the classic energy methods for stability. ${ }^{52} \mathrm{~A}$ study of the stability of rigid body pairs using the energy-momentum method is directly relevant to the evolution of binary asteroids and the dynamic environments they encounter through their lifetime. The current literature, however, uses only the Arnold or energy-Casimir methods, ${ }^{57}$ which generally give weaker results. It would be very interesting to make use of the full block diagonalization power of the energy-momentum, which has proven useful in related problems. ${ }^{2,7}$ In addition, there is a converse to the method that allows one to deduce the development of instabilities due to forcing and dissipation, ${ }^{29,30}$ which can have important long-term effects on the dynamics. ${ }^{62}$ 


\section{Symmetries of the Rigid Bodies}

If, in addition, the individual rigid bodies exhibit configuration symmetries of their own, there are additional symmetries acting on the right. Since these commute with the symmetry of $S O(3)$ acting on the left, one can again make use of reduction by stages.

\section{Example: Spherical Symmetry}

If the first body is spherical, and the second body has no symmetry, the additional symmetry group is $S O(3) \times\{e\}$ acting on the right. On the Hamiltonian side, the reduced space is then $T^{*} \mathbb{R}^{3} \times \mathfrak{S e}(3) \times \mathfrak{G D}(3)^{*}$. The $T^{*} \mathbb{R}^{3}$ terms correspond to position and linear momentum of the center of mass of the first rigid body, in the body frame of the second rigid body. The $\mathfrak{S e}(3)^{*}$ term involves the spatial linear and angular momentum of the second rigid body, and $\mathfrak{S D}(3)^{*}$ involves the body angular momentum of the first rigid body. There is a similar picture on the Lagrangian side.

\section{Example: Cylindrical Symmetry}

If the first body is cylindrical and the second body has no symmetry, the additional symmetry group is $S^{1} \times e$ acting on the right. The reduced space is then $T^{*}\left(S^{2} \times \mathbb{R}^{3}\right)$ $\times \mathfrak{s e}(3)^{*} \times \mathbb{R}$. The $T^{*}\left(S^{2} \times \mathbb{R}^{3}\right)$ terms correspond to the orientation of the symmetry axis and the position of the first rigid body, in the body frame of the second rigid body, as well as the conjugate momenta. The $\mathfrak{s e}(3)^{*}$ term involves the spatial linear and angular momentum of the second rigid body and the $\mathbb{R}$ term involves the component of the body angular momentum of the first rigid body in the direction of its symmetry axis.

The preceding reduction procedures give a global description of the reduced spaces and dynamics. This is important, since ad hoc choices for coordinate systems that are only locally valid can introduce significant computation overheads if it is constantly necessary to switch between local charts in the course of a long time simulation. The variational, Poisson, and symplectic structures on these reduced spaces can be constructed systematically from the general theory of reduction, and, as we have indicated, this is particularly useful in classifying a hierarchy of simplified models.

Relative equilibria and relative periodic orbits correspond to equilibria and periodic orbits in the shape space $Q / G$. The bifurcation of a relative equilibria into a relative periodic orbit through a Hamiltonian Hopf bifurcation in the shape space is also particularly interesting for the study of real world objects that may be subject to parametric uncertainty, and the effects of such parameter dependence need to be studied in our models. More generally, the study of these phase space objects, their stability, and their bifurcation patterns can be used to characterize motion in different regions of phase space. ${ }^{63}$

The phenomena of pinch points in the dynamics could possibly be related to the presence of resonances corresponding to discrete symmetries, or possibly to degenerate values of the momentum map corresponding to the non-free action of a continuous symmetry group. It is well known that pinch points of this sort are related to reduction in singular cases (an example is given in, for instance, Ref. 64). An example of a problem with such a non-free action is the double spherical pendulum, and the bifurcation from the degenerate downward configuration into relative 
equilibria and relative periodic orbits has been analyzed using the machinery of bifurcation theory and singular reduction theory. ${ }^{65} \mathrm{~A}$ better understanding of the pinch points observed in dynamical systems will be directly applicable in identifying the regions of phase space that contribute to statistical transport phenomenon.

\section{Geometric Phases}

The issue of geometric phases is not only of interest in the dynamics of an asteroid pair, but is also relevant to the problem of landing a spacecraft on a spatially extended and irregular asteroid (as in the recent NEAR mission to the asteroid EROS). An understanding of geometric phases allows one to relate the motion of internal rotors and their effect on the relative orientation of the spacecraft with respect to the asteroid. This provides a precise method for controlling the relative orientation, which is in contrast to the use of microthrusters, which introduce torque that needs to be compensated for once the desired orientation is achieved. This same approach can be applied to the study of the tidally locked Pluto-Charon system and may help address some aspects of the current controversy surrounding the value of the orbital eccentricity of Charon and how it arose. ${ }^{66}$

Geometric phases can also be applied to spacecraft control in close proximity to an asteroid. ${ }^{67}$ In this context of control, the method of controlled Lagrangian and Hamiltonian systems may be a useful tool (see Ref. 68 and citations therein). This is generalization of potential and kinetic shaping techniques that has been applied in the context of spacecraft dynamics to modify the Poisson structure of the system using feedback control laws. A corresponding theory for the reduction of systems with symmetry also exists, using the method of Lagrangian and Hamiltonian reduction.

\section{PHASE SPACE STRUCTURES}

\section{Global Theory of Chaotic Transport}

After systematically categorizing the systems of interest using geometric mechanics, the next step is to use efficient computations guided by dynamical systems theory to explore statistical questions. These questions, including the probability of binary pair formation and subsequent escape or impact, and ejecta redistribution around a rotating small body can be cast as phase space transport problems. The systems of interest, being Hamiltonian in nature, likely have a global mixed phase space structure of stable and chaotic zones; that is, islands of KAM tori and a "chaotic sea" between them. A semi-analytical global theory of chaotic transport is emerging that combines the theory and numerics of lobe dynamics and tube dynamics. ${ }^{54,69,70}$ The lobe dynamics techniques come partly from fluid dynamics-for example, those developed in the last two years using the MANGEN software-providing unprecedented long-term precision calculations. ${ }^{71,72}$

\section{Efficient Computation of Statistical Quantities}

This global theory allows one to tackle transport problems by focusing on the objects that are important for transport. Dynamical systems theory has been used previously to give a qualitative description of the topological features of the phase 
space in certain reduced F2BP models, such as periodic orbits, resonance regions, and chaotic zones. One can make this analysis more quantitative by computing statistical measures, such as residence times within regions of phase space, transport rates between various regions, and the overall level of mixing between regions.

Since the F2BP is such a fundamental model in studying small body orbital and rotational dynamics, the present context should shed light on several interesting and important problems. Specifically, it may help address the problem of slowly rotating asteroids,${ }^{50}$ it will be able to quantify the rate at which asteroid binaries are disrupted due to their mutual interactions following their creation, ${ }^{25}$ and the important issue of chaotic planetary obliquities and their probability of transition to different states. ${ }^{37}$

\section{A SIMPLIFIED MODEL FOR BINARY ASTEROIDS}

\section{The Restricted Full Two-Body Problem (RF2BP)}

The simplest model that exhibits the basic ejection and collision dynamics we are interested in studying is given by the RF2BP, which is a simplification of Equation (1) in the limit $\mu \rightarrow 0$. The equations of motion for the massless particle in a rotating Cartesian coordinate frame and appropriately normalized are

$$
\begin{aligned}
& \ddot{x}-2 \dot{y}=-\frac{\partial U}{\partial x} \\
& \ddot{y}+2 \dot{x}=-\frac{\partial U}{\partial y},
\end{aligned}
$$

where

$$
\begin{aligned}
U(x, y) & =-\frac{1}{\sqrt{x^{2}+y^{2}}}-\frac{1}{2}\left(x^{2}+y^{2}\right)+U_{22} \\
U_{22} & =-\frac{3 C_{22}\left(x^{2}-y^{2}\right)}{\left(x^{2}+y^{2}\right)^{5 / 2}} .
\end{aligned}
$$

The system (3) has one free parameter, the gravity field coefficient $C_{22}$, commonly termed the "ellipticity", that varies between 0 and 0.05 for physical systems. The system (3) may be readily derived from Lagrangian mechanics using the method of "moving systems" 27 and, therefore, has an energy integral (a Jacobi integral), namely

$$
E=\frac{1}{2}\left(\dot{x}^{2}+\dot{y}^{2}\right)+U
$$

This system has four equilibrium points symmetrically placed along the $x$ and $y$ axes, each at a radius of $R \sim 1+\ldots$, with the higher order terms arising from the $U_{22}$ potential. In general, we do not consider motion at radii much less than $R \sim 1$, since these trajectories usually impact on the central body. Furthermore, note that these equations have very bad behavior as $R \rightarrow 0$, which is another reason to avoid radii much inside the equilibrium points.

The region of space where $R>1$ is very interesting, however. First, there are continuous families of near-circular periodic orbits that exist for any given radius. Occasionally, these periodic orbits are resonant with the rotation rate of the system, and 
at these points we can find additional families of "elliptical" periodic orbits that branch off.

\section{Realms and Regions in the RF2BP}

In the RF2BP, the value of the energy $E$ indicates the type of global dynamics possible. For example, for energies above a threshold value, (i.e., $E>E_{S}$, where $E_{S}$ corresponds to the energy of symmetric saddle points along the $x$-axis), movement between the realm near the asteroid (interior realm) and away from the asteroid (exterior realm) is possible, as in FIGURE 3 A. This motion between realms is mediated by phase space tubes, to be described shortly. For energies $E \leq E_{S}$, no such movement is possible. Within each realm, the phase space on appropriately chosen Poincaré sections is organized further into different regions, connected via lobes.

\section{Tube Dynamics: Transport between Realms}

In Figure $3 \mathrm{~A}$ the interior and exterior realms are linked by tubes in phase space, bounded by the stable and unstable invariant manifolds associated with periodic orbits around the symmetric saddle points. The role these tubes play in global transport between realms is referred to as tube dynamics. ${ }^{54}$ On each three-dimensional
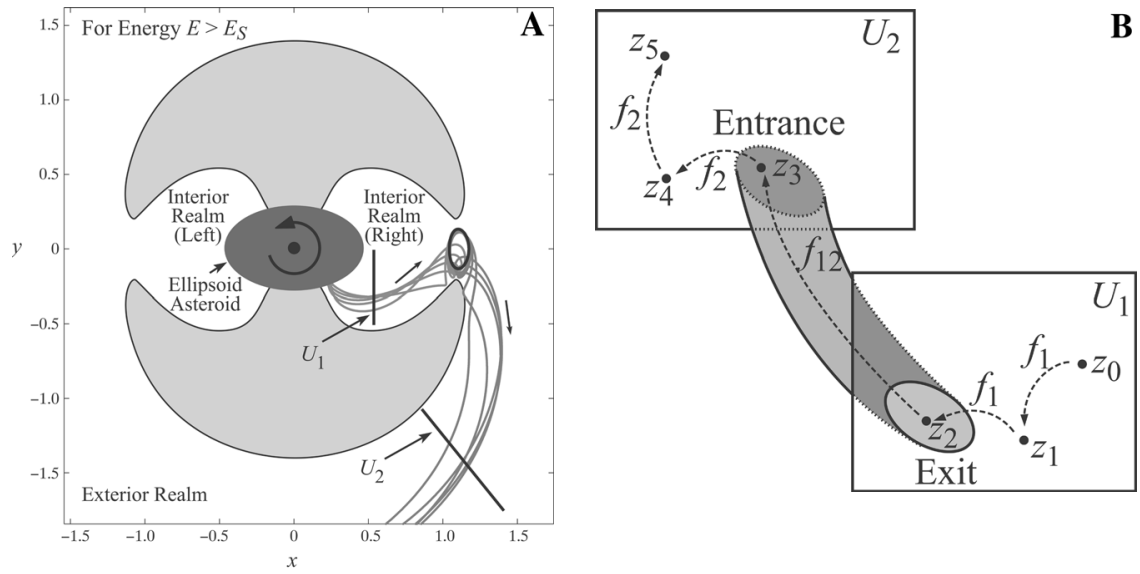

FIGURE 3. Planar restricted F2BP with uniform rotation: realms and allowable motion. (A) For energies, $E$, above a threshold, $E_{S}$, movement is possible between the realm near an asteroid and away from the asteroid. In this rotating frame, the $x$-axis coincides with the long axis of the elliptical asteroid. The origin is shown as a large black dot at the center of the asteroid. The value of the "ellipticity", $C_{22}$, is 0.05 . Varying this parameter changes the shape of the asteroid and the subsequent potential through the $U_{22}$ term in Equation (3). (B) Poincaré sections in various realms-in this case in the interior and exterior realms, $U_{1}$ and $U_{2}$, respectively-are linked by phase space tubes that live in surfaces of constant energy, Equation (4). Under the Poincaré map $f_{1}$ on $U_{1}$, a trajectory reaches an exit, the last Poincaré cut of a tube before it enters another realm. The map $f_{12}$ takes points in the exit of $U_{1}$ to the entrance of $U_{2}$. The trajectory then evolves under the action of the Poincare map $f_{2}$ on $U_{2}$. 
energy surface these two-dimensional tubes partition the surface, acting as separatrices for the flow through the bottleneck regions around the saddle points: particles inside the tubes move between realms, and those outside the tubes not. For example, an ejecta particle liberated from the surface of the asteroid into the exterior realm with an energy just above the threshold can impact the asteroid only by passing through one or the other of the pair of exterior branch stable manifold tubes associated with the two periodic orbits around the saddle points (the right periodic orbit is shown in FIG. $3 \mathrm{~A}$ ).

The projections of these tubes onto the configuration space appear as strips and trajectories on the tubes wind around them. A few trajectories on a couple of tubes are shown in Figure 3 A. On the Poincare section, the last Poincaré cut of the stable manifold tube is called the exit, because points there exit the interior realm and go to the exterior realm. The time reverse situation holds for an entrance, and when a particle goes through the exit in one realm, it must enter the entrance of another.

These transition dynamics are of direct interest to the evolution of a dissipating binary system, since it may experience several junctures when it can transition between a state close to its final equilibrium and a state where more dynamic evolution is possible. Similarly, the long-term variation of planetary obliquities may be modeled using this approach.

\section{Lobe Dynamics: Transport between Regions}

Tubes are only one part of the global transport picture. The study of transport between regions within a particular realm can be reduced to the study of an associated Poincaré section in that realm. Following Reference 73, lobe dynamics theory states that the two-dimensional phase space $M$ of the Poincaré map $f$ can be partitioned into disjoint regions of interest, $R_{i}, i=1, \ldots, N_{R}$, such that $M=\cup R_{i}$ (see FIGURE 4A). The boundaries between regions are pieces of stable and unstable manifolds of hyperbolic fixed points, $p_{i}, i=1, \ldots, N$. Moreover, transport between regions of phase space can be completely described by the dynamic evolution of turnstile lobes enclosed by segments of the stable and unstable manifolds (FIG. 4B).

To keep track of points as they move between regions, suppose that, at $t=0$, region $R_{i}$ is uniformly covered with points of species $S_{i}$. The transport problem becomes one of describing the distribution of species $S_{i}$ throughout the regions $R_{j}$ for any time $t=n>0$. Two quantities of interest - the flux of species $S_{i}$ into region $R_{j}$ on the $n$th iterate, $\alpha_{i, j}(n)$, and the total amount of species $S_{i}$ in region $R_{j}$ just after the $n$th iterate, $T_{i, j}(n)$ - can be expressed compactly in terms of intersection areas of images or pre-images of turnstile lobes. In our application, the Poincaré map of a reduced F2BP model possesses resonance bands consisting of alternating unstable and stable periodic points. For instance, we can study the transport from stable configurations to escape configurations or the transport into and out of spin-orbit resonances, and many other transport questions that have implications for the current state of bodies in the solar system.

\section{Computational Tools for Lobe Dynamics}

Although lobe dynamics has always been recognized as an exact transport theory and can theoretically give short- and long-term transport rates, computation issues 

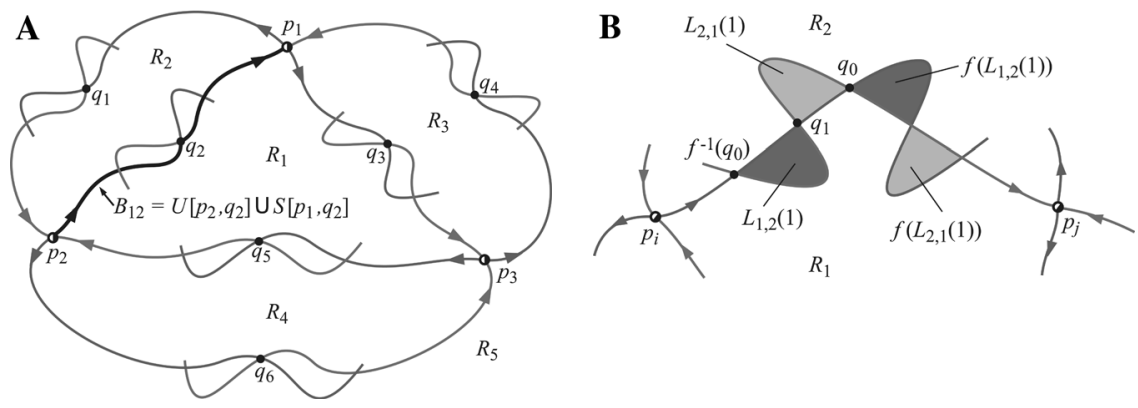

FIGURE 4. Transport between regions in phase space. (A) A point $q_{k}$ is called a primary intersection point (pip) if $S\left[p_{i}, q_{k}\right]$ intersects $U\left[p_{j}, q_{k}\right]$ only at the point $q_{k}$, where $U\left[p_{j}, q_{k}\right]$ and $S\left[p_{i}, q_{k}\right]$ are segments of the unstable and stable manifolds, $W^{u}\left(p_{j}\right)$ and $W^{s}\left(p_{i}\right)$, respectively, of unstable fixed points of the Poincaré map $f$. The union of segments of the unstable and stable manifolds form partial barriers, or boundaries $U\left[p_{j}, q_{k}\right] \cup S\left[p_{i}, q_{k}\right]$ between regions of interest $R_{i}, i=1, \ldots, N_{R}$, in $M=\cup R_{i}$. The region on one side of the boundary $B_{12}$ is labeled $R_{1}$ and the other side labeled $R_{2}$. (B) Let $q_{0}, q_{1} \in W^{s}\left(p_{i}\right) \cap W^{u}\left(p_{j}\right)$ be two adjacent pips; that is, there are no other pips on $U\left[q_{0}, q_{1}\right]$ and $S\left[q_{0}, q_{1}\right]$, the segments of $W^{u}\left(p_{j}\right)$ and $W^{s}\left(p_{i}\right)$ connecting $q_{0}$ and $q_{1}$. The region interior to $\mathrm{U}\left[q_{0}, q_{1}\right] \cup S\left[q_{0}, q_{1}\right]$ is a lobe. Then $S\left[f^{-1}\left(q_{0}\right), q_{0}\right] \cup U\left[f^{-1}\left(q_{0}\right), q_{0}\right]$ forms the boundary of two lobes; one in $R_{1}$, labeled $L_{1,2}(1)$, and the other in $R_{2}$, labeled, $L_{2,1}(1)$. Under one iteration of $f$, the only points that can move from $R_{1}$ into $R_{2}$ by crossing $B_{12}$ are those in $L_{1,2}(1)$ and the only points that can move from $R_{2}$ into $R_{1}$ by crossing $B_{12}$ are those in $L_{2,1}(1)$. The union of the two lobes, $L_{1,2}(1) \cup L_{2,1}(1)$, is called a turnstile.

have limited its applications. ${ }^{74,75}$ The manifolds computed in such problems are typically rather convoluted, as implied in FIGURE 4. Furthermore, the length of these complicated curves grows quickly with the size of the time window of interest. The number of points needed to describe long segments of manifolds can be prohibitively large if brute force approach computation methods are used.

Recent efforts made to incorporate lobe dynamics into geophysical and chemical transport calculations have brought new techniques to compute invariant manifolds. ${ }^{70-72,76,77}$ We have been able to compute long segments of stable and unstable manifolds with high accuracy by conditioning the manifolds adaptively; that is, by inserting more points along the manifold where the curvature is high. As a result, the length and shape of the manifold is not an obstacle and many more iterates of lobes than hitherto possible can be generated accurately. Initial tests show that this method of computing lobes using MANGEN is very relevant to the problems we investigate.

\section{Phase Space Structure in the Exterior Region}

A Poincaré surface-of-section (s-o-s) of the RF2BP at an energy just above the threshold where the bottleneck appears (i.e., $E>E_{S}$, the case in FIGURE $3 \mathrm{~A}$ ) illustrates the relevance of tube and lobe dynamics in this system. In FigurE 5, the s-o-s was taken in the exterior region along the positive $x$-axis. At the energy chosen, there is a bottleneck around the equilibrium points along the $x$-axis. Particles beginning in the exterior region reach the interior region and subsequently collide with the asteroid 
if they lie within the phase space tubes associated with the unstable periodic orbits about either the left or right saddle points. Thus, we refer to the tube slices on this $\mathrm{s}-\mathrm{O}-\mathrm{S}$ as tube slices of collision. Furthermore, particles are ejected from the system if they lie within lobes enclosed by the stable and unstable manifolds of a hyperbolic fixed point at $(+\infty, 0)$, referred to as lobes of ejection.

Physical insight is gained into the conditions for ejection by transforming to Delaunay variables. In FIGURE 5B, the semimajor axis is shown versus the argument
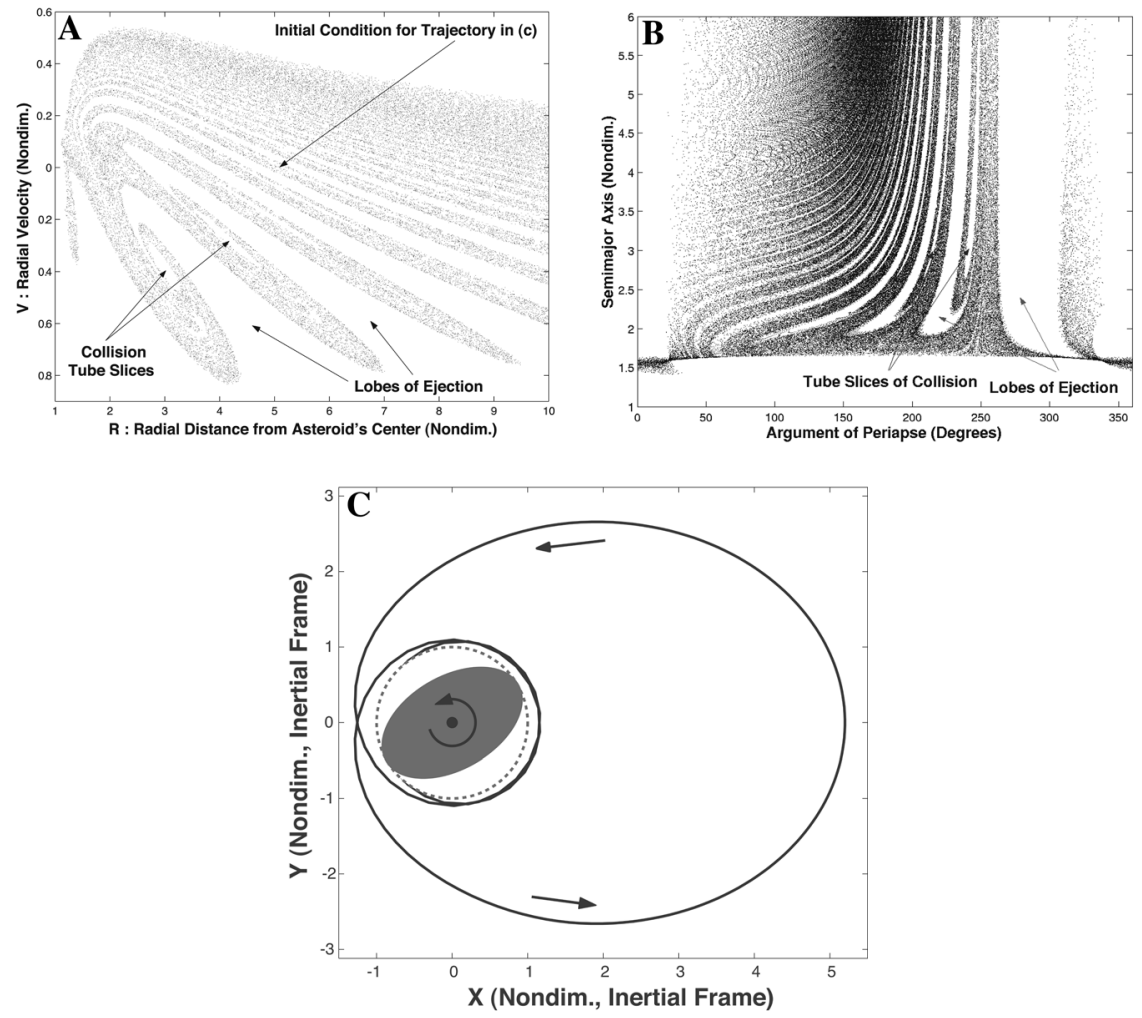

FIGURE 5. Mixed phase space structure for the restricted model: the dynamics of particle ejection from, and collision with, a distended asteroid. A Poincaré surface-of-section taken in the exterior region $(R>1)$. (A) Particles not ejected after 15 iterates are shown. The coordinates are the radial velocity of the particle versus its radial distance from the asteroid center. The finger-like structures visible here, the lobes of ejection, have been seen in some chemistry problems. ${ }^{70,78}$ (B) Semi-major axis versus argument of Periapse with respect to the rotating asteroid (the body-fixed frame). (C) An interesting trajectory corresponding to the labeled point on the Poincare section in (A) is shown. The trajectory escapes the asteroid only to fall back upon it after one large elliptical orbit. The units are in terms of the semiaxis length of the asteroid, which is shown schematically at one instant of time. The asteroid is rotating counterclockwise around its center (shown as a large dot at the origin). The long axis of the asteroid sweeps out the circular region bounded by the dashed line. 
of periapse with respect to the rotating asteroid (the body-fixed frame). The alternate fates of collision and ejection are seen to be intimately intermingled in the phase space. Note that the number of particles remaining in the fourth quadrant $\left(270^{\circ}-360^{\circ}\right)$ is smaller than that in the other three quadrants, in agreement with observations made elsewhere. ${ }^{16}$ FIGURE $5 \mathrm{C}$ shows a particular trajectory that escapes from the asteroid only to fall back upon it, a peculiar phenomenon encountered in tube dynamics. ${ }^{54}$

In the RF2BP, we are considering the motion of a massless particle; that is, the limit $\mu \rightarrow 0$. The next step in our study is to assume that the particle has mass; that is, $\mu>0$ in Equation (1). Due to the gravitational attraction of the particle, the rotation of the asteroid is now non-uniform and must be tracked. Although this adds another dimension to the phase space, it also adds a new integral, the angular momentum $\mathcal{K}$ in Equation (2). Thus, we can still use the two-dimensional Poincaré map analysis, as in FigURE 5. We are currently in the process of characterizing the global phase space structure that gives rise to the complex behavior seen in FIGURE 2 and similar problems.

\section{CONCLUSIONS AND FUTURE DIRECTIONS}

We have outlined the general setting for the application of techniques from geometric mechanics and dynamic systems transport calculations to full-body problems (FBPs). General FBPs have a wide range of other interesting aspects as well, including the 6-DOF guidance, control, and dynamics of vehicles, the dynamics of interacting or ionizing molecules, the evolution of small body, planetary, or stellar systems, and almost any other problem where distributed bodies interact with each other or with an external field.

This paper focuses on a motivating example of a full two-body problem from dynamical astronomy - the problem of asteroid pairs and the calculation of binary asteroid escape rates. We have given some preliminary results for a simplified model of binary asteroid escape rates, describing how lobe and tube dynamics lead to a rich phase space even for this simplified system.

Some future directions for this area of research are as follows.

\section{Variational Integrators for FBPs}

As is noted in the literature, the Poisson structures that are obtained in the reduced models are non-canonical and, as such, applying standard symplectic algorithms will not provide the long-time stability we have come to associate with such numerical methods, since they preserve the canonical symplectic form, which is not consistent with the reduced dynamics. Instead, we make use of reduction theory for discrete systems, the abelian Routh case of which is worked out and applied to the $J_{2}$ problem (satellites in orbit about an oblate planet). ${ }^{79}$ We will study transport phenomena in the F2BP using numerical schemes that preserve the geometric structures inherent in the system. Toward this end, we will redo the above computations for other F2BP cases and carry out long time accurate time integrations using variational integrators that capture transport rates and the detailed structure of chaotic sets. 
Variational integrators provide a systematic and powerful extension of symplectic integrators that have a proven track record in celestial mechanics for long-term integrations; see, for instance References 31,32 , and 80 . The variational setting allows for extensions to partial differential equations, for asynchronous methods, as well as the use of symmetry methods that are central to this approach.

The idea of the variational integration method is to discretize Hamilton's principle directly rather than discretize the associated Euler-Lagrange equations. The discrete form of Hamilton's principle then determines the numerical algorithm for that system. The accuracy to which the approximation of Hamilton's principle is done is reflected in the accuracy of the algorithm itself; however, much more is true. When integrators are designed this way, they have remarkable respect for the geometric mechanics aspects of the problem, including excellent energy behavior, conservation of the symplectic structure and exact conservation of a discrete version of the Noether quantities associated with symmetries. It is perhaps surprising that these methods work well even for dissipative and forced systems. The idea in this case is to discretize the Lagrange-d'Alembert principle rather than Hamilton's principle.

Recent references develop and document the success of this methodology. ${ }^{33,53,81}$ FIGURE 6 shows a computation for a particle moving in the plane under a radially symmetric polynomial potential, without and with a small amount of friction. The exact preservation of the conserved quantities, in this case energy, is a natural consequence of the discrete variational principle. In addition, FIGURE 6 illustrates that these methods can handle dissipative systems and get the energy decay rate accurate as measured against a benchmark calculation. ${ }^{82}$ In addition, it is shown ${ }^{33,83}$ that many statistical quantities, such as temperature and the structure of chaotic invariant sets, are accurately captured by variational integrators.

A key feature is the development of asynchronous variational integrators (AVIs), ${ }^{33}$ which allow one to take different time steps at various spatial points and yet retain all the beautiful structure preserving properties of variational integrators. This is important in FBPs since the various bodies may have vastly different spatial and temporal scales, and so one must adapt the time steps according to these different scales. The AVI approach is a natural way to study multiscale dynamics without sacrificing structure preservation. Interestingly, this is closely related to methods used in molecular dynamics (see the work of Barash and Schlick ${ }^{84}$ ).

\section{Reduction for Discrete Mechanics}

There has been significant work done on reduction theory for discrete variational mechanics. An example, developed in Reference 79 is that of the dynamics of a satellite in the presence of the bulge of the Earth, in which many interesting links with geometric phases and computation in the reduced and unreduced spaces are noted. However, for the F2BP, this basic theory needs to be generalized, a key point being the extension to the case of nonabelian symmetry groups and the generalization beyond the Euler-Poincaré context. ${ }^{85}$ We intend to undertake such a study and to apply it to the case of the F2BP. There is a cautionary message in the work that numerical experience shows-it is important to fully understand the reduction of discrete variational mechanics, since applying standard numerical algorithms to the reduced equations obtained from continuous reduction theory may not yield the desired results and long term stability may not be respected. A related goal is to 

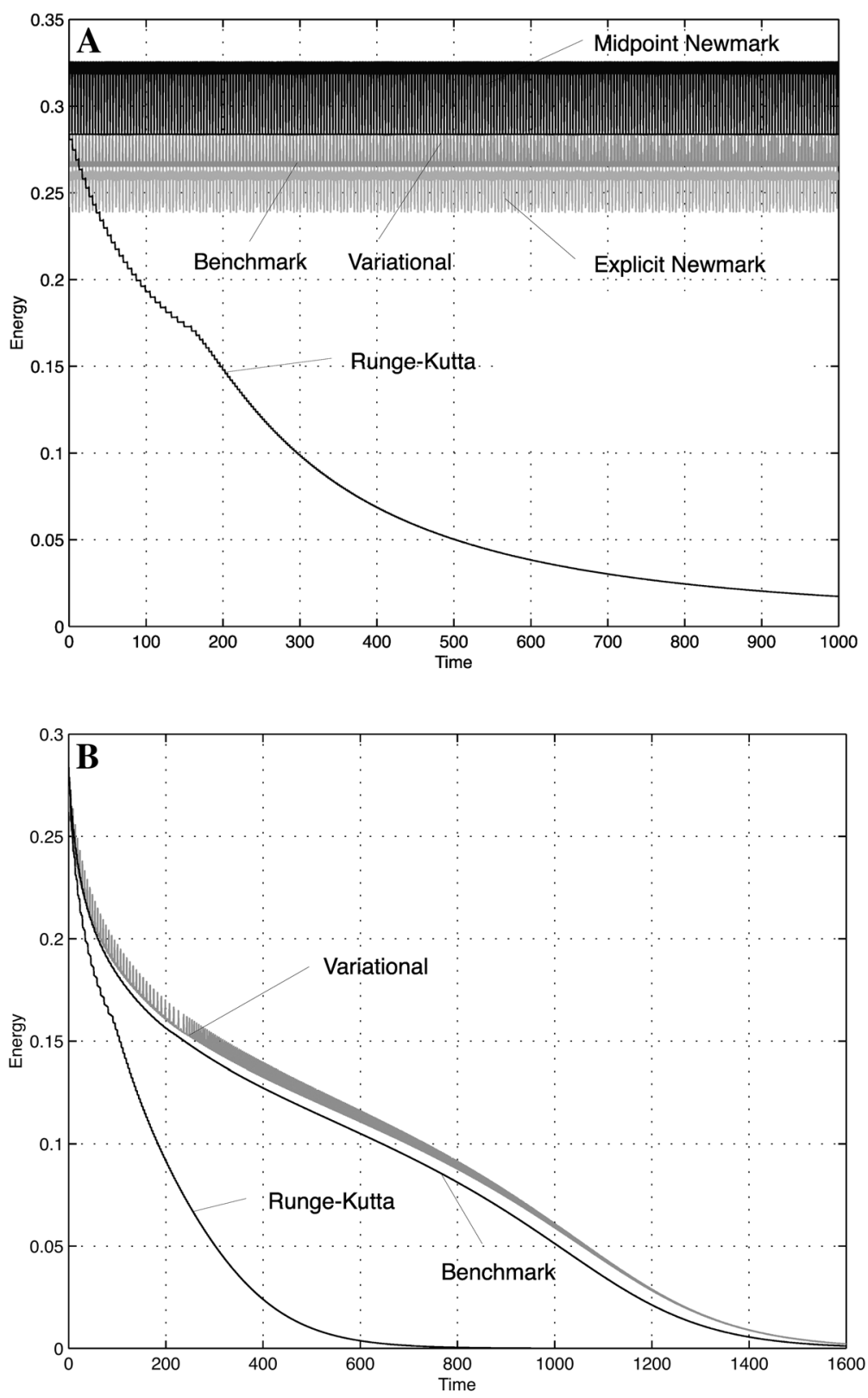

FIGURE 6. Conservative system (A) and dissipative system (B). 
combine variational integrators and adaptive manifold conditioning to give a state of the art package to compute manifolds, lobes, and turnstiles in a variety of systems, including the F2BP. The use of variational integrators is expected to increase the accuracy of computation of long term statistical quantities, such as transport rates. We also may combine these techniques with those of the Dellnitz group using box elimination methods. 36,86

\section{The Full N-Body Problem}

A natural extension of the F2BP is the problem of multiple distributed bodies interacting with each other, the full $N$-body problem. For example, it would be interesting to put the work of Reference 20 into a modern context using the same reduction and simulation theory outlined above. Recent success in applying classical $\mathrm{N}$-body problem results to the $\mathrm{F}_{2} \mathrm{BP}^{22,25}$ indicates that progress can indeed be made in developing this approach.

\section{Incorporation of Deformation and Dissipation Effects}

The incorporation of deformation and dissipation effects into one or both of the bodies provides an important path toward understanding full-body dynamics in a deeper way. Even if the simplest deformation and dissipation models are incorporated, based on small deformation and linear dissipation assumptions, these effects can have major influence on full-body attitude and orbital dynamics. This phenomenon is well known in planetary dynamics ${ }^{87}$ and has also been characterized in the field of space structure dynamics and control.

Models that incorporate attitude, translation, and finite deformation degrees of freedom for a distributed body in a central body gravitational field, have been developed (e.g., see Ref. 88). Even in this relatively simplified version of a FBP, the presence of deformation and dissipation can provide mechanisms for energy transfer between translation and attitude. They also play important roles in controlled FBPs, where physical or control effects directly influence the deformation and dissipation, and indirectly influence attitude and/or translation.

It is also of interest to extend recent work on the stability and control of satellites with flexible appendages to describing the natural dynamics of deformable bodies in orbit about a rigid body. As examples, the stability and Hamiltonian structure of a rigid body with attached flexible rod was analyzed. ${ }^{1}$ More recently, ${ }^{89}$ induced instabilities in a satellite with momentum wheels with an attached string was considered. This "radiation induced instability" is related to the dissipation induced instabilities. ${ }^{29,30}$ Such instabilities can occur when there is a saddle point in the energy-momentum function but all eigenvalues of the linearized system lie on the imaginary axis, situations that occur in the relative equilibria of many FBPs. These results, when coupled with a recent rediscovery of an elastic solution for triaxial ellipsoids, ${ }^{90}$ could yield significant enhancements in our current understanding of tidal disruptions and dissipation in small body systems. ${ }^{91,92}$

\section{Astronomy and Planetary Science}

Generalized models of the F2BP have many applications in the field of astronomy and planetary science. In the following we mention a few specific targets for our 
research, chosen as good candidates for the application of our systematic approach to full-body problems.

An important outcome of this phase of analysis could involve the long-term simulation of the effects of the Yarkovsky force on the translational and rotational motion of asteroids. This effect has recently been recognized to be an important component in describing the evolution and current state of the solar system. Although much work has been done on the modeling and prediction of its effects, ${ }^{39-42}$ long-term simulations of its effects using realistic models is currently not possible. The heart of the difficulty is the delicate relation between the rotation state of an asteroid and the net result of the Yarkovsky force (due to thermal inertia in the body). The suite of simulation tools we are developing will be able to directly address this question and would unquestionably advance our ability to model and understand this important effect.

A related open question in planetary science is an explanation for the large number of slowly rotating asteroids. ${ }^{50,93}$ Current distribution statistics cannot be accounted for by using the traditional theory of asteroid rotation state evolution. Although Yarkovsky forces have been considered as a possible explanation, ${ }^{42}$ a strong case has not yet been built. Mutual disruption of binaries under their own gravitational interaction is another potential mechanism for the creation of slowly rotating and tumbling asteroids. To properly address whether this can account for the noted excess of slow rotators, a deeper study of ejection probability in binary asteroids must be made, starting from the work described in References 23, 25, and 50, coupled with an analysis of the likely formation energies of these binaries, drawing on recent work in this area. ${ }^{92,94-98}$

Related to this study is a complete analysis of the formation and evolution of a binary asteroid or Kuiper belt object, including the effect of energy dissipation and external force perturbations. The simulation tools being developed for the F2BP will be ideal for the simulation of these systems over long time spans, allowing for the development and testing of various hypotheses and constraints on the life of these objects. Based on current theoretical models and results $23,25,99$ a binary will be subject to mutual disruption or impact during its evolution, and only a fraction of binary bodies should survive into a long-term stable state. The resolution and understanding of this question is completely open, but would be naturally addressed using a FBP approach. As part of this analysis, we will also examine the Pluto-Charon system, currently subject to controversy based on the apparent eccentricity of their mutual orbit. 66

Recent reanalysis of the long-term evolution of comet nucleus rotation states accounting for realistic models of outgassing torques on the bodies has shown a remarkably rich set of steady-state states for their rotation states. ${ }^{43}$ Direct simulation of these effects over long-periods is currently not possible, but may be attainable from the outcome of our work. Furthermore, the non-trivial dependence on rotation state on the heliocentric orbit of a comet invites a more complete analysis of the coupling of nucleus rotation and its orbital variation due to outgassing, which is the classical problem of comet nucleus orbit mechanics. ${ }^{44}$

An additional, novel idea is to apply the F2BP, with dissipation and deformation effects, to describing the interaction of two or more distributed galaxies. As a first step, this work would link the classical theory of ellipsoidal figures of equilibrium ${ }^{100}$ 
with the effect of a non-collision interaction between two galaxies. Following such an encounter, each of the distributed bodies would feel a near-impulsive change in their energy and angular momentum, due to exchanges between the distributed bodies. $^{24}$ Treating these stellar collections as FBPs could lead to fruitful new insights and approaches to describe their evolution and dynamics.

\section{Vehicle Dynamics and Control}

Control problems arise in FBPs where one of the bodies is an artificial body, such as a controlled spacecraft or satellite. There is a vast literature on attitude control and orbit control of spacecraft and the success of most space missions has depended on this capability. With only few exceptions, this literature treats attitude control problems and orbit control problems as independent problems. This is reflected in the organization chart of most space agencies and space industries; attitude control groups and orbit control groups operate independently.

The thesis of this area of research, based on the fundamental definition of FBPs, is that attitude and orbit dynamics and control problems should be treated in a unified way. A consequence of this thesis is that control problems for FBPs should be formulated, analyzed, and implemented using prior knowledge of the full-body dynamics. Geometric properties, such as symmetries and reduction, are not only important for analysis of full-body dynamics but are crucial to full-body control as well. Most full-body control problems are likely to be nonlinear and to require special methods for control design and analysis that are tailored to the full-body dynamics.

Nonlinear control methods should provide excellent frameworks for studying full-body control problems. These methods include controlled Lagrangian, ${ }^{101}$ geometric phases, ${ }^{10,61}$ and differential geometric approaches. ${ }^{10,102}$ These approaches have been developed and successfully applied to the control of rigid bodies and multibody systems, taking into account motion constraints, symmetries, controlactuation assumptions, deformation degrees of freedom, and dissipation. They have been used to construct controllers for specific rigid-body and multibody spacecraft attitude control problems using thrusters, reaction wheels, proof mass actuators, appendages, and tethers. By analyzing and describing these systems from a more general vantage, it is often possible to gain additional insight into the known symmetries of the simpler problem, and potentially to discover new symmetries not appreciated before. A similar philosophy can be applied to a reanalysis of vehicle motion in fluid fields, where the existence of the fluid can be idealized as the limiting model of a second body interaction on the vehicle. Because these vehicles have strong coupling between rotational and translational motion and are considered to be extremely difficult analytical problems, any advance in understanding such vehicles would be important.

\section{Chemical Dynamics}

The mathematical description of transport phenomena applies to a wide range of physical systems across many scales. ${ }^{73,103,104}$ The recent and surprisingly effective application of methods combining dynamical systems ideas with those from chemistry to the transport of Mars ejecta by several of the coauthors in collaboration with chemists, underlines this point. ${ }^{35}$ Thus, techniques developed to study transport in 
the solar system are truly fundamental and broad-based. The methods may be applied to diverse areas of study, including fluid mixing ${ }^{71,76,105-109}$ and $N$-body problems in physical chemistry. ${ }^{110} \mathrm{~A}$ basic framework for this theory has already allowed several of the coauthors to develop a new low-fuel mission concept to explore the moons of Jupiter. ${ }^{111}$ Any improvement made in the software in the course of the work outlined above can, thus, be applied to areas beyond dynamic astronomy.

\section{ACKNOWLEDGMENTS}

This research was partially supported by a Max Planck Research Award and NSF ITR grant ACI-0204932. The work described in this paper was carried out in part at the Jet Propulsion Laboratory, California Institute of Technology, under contract with NASA.

\section{REFERENCES}

1. Krishnaprasad, P.S. \& J.E. Marsden. 1987. Hamiltonian structure and stability for rigid bodies with flexible attachments. Arch. Rat. Mech. Anal. 98: 137-158.

2. Krupa, M., M. Schagerl, A. Steind, et al. 2001. Relative equilibria of tethered satellite systems and their stability for very stiff tethers. Dyn. Syst. 16: 253-278.

3. ScheERES, D.J. 2004. Stability of relative equilibria in the full two-body problem. Ann. N.Y. Acad. Sci. 1017: this volume.

4. WisDOM, J. 1987. Rotational dynamics of irregularly shaped natural satellites. Astron. J. 94: $1350-1360$.

5. Touma, J. \& J. Wisdom. 1994. Evolution of the Earth-Moon system. Astron. J. 108: 1943-1961.

6. BECK, J.A. \& C.D. HALL. 1998. Relative equilibria of a rigid satellite in a circular keplerian orbit. J. Astronaut. Sci. 46: 215-247.

7. WANG, L.S., P.S. KRishnaprasad \& J.H. MADdOCKS. 1991. Hamiltonian dynamics of a rigid body in a central gravitational field. Celest. Mech. Dyn. Astron. 50: 349-386.

8. WANG, L.S., P.S. KRISHNAPRASAD \& J.H. MADDOCKS. 1992. Steady rigid-body motions in a central gravity field. J. Astronaut. Sci. 40: 449-478.

9. WANG, L.S., K.-Y. LiAn \& P.-T. ChEN. 1995. Steady motions of gyrostat satellites and their stability. IEEE Trans. Auto. Cont. 40: 1732-1743.

10. Rui, C., I. Kolmanovsky \& N.H. McClamroch. 2000. Nonlinear attitude and shape control of spacecraft with articulated appendages and reaction wheels. IEEE Trans. Auto. Cont. 45: 1455-1469.

11. Shen, J. \& N.H. McClamroch. 2001. Translational and rotational spacecraft manuevers via shape change actuators. In Proc. 2001 American Control Conference, 3961-3966.

12. Bloch, A.M., P. Crouch, J.E. Marsden \& T.S. Ratiu. 2002. The symmetric representation of the rigid body equations and their discretization. Nonlinearity 15: 13091341.

13. Dobrovolskis, A.R. \& J.A. Burns. 1980. Life near the roche limit: behavior of ejecta from satellites close to planets. Icarus 42: 422-441.

14. Chauvineau, B., P. Farinella \& F. Mignard. 1993. Planar orbits about a triaxial body: applications to asteroidal satellites. Icarus 105: 370-384.

15. SCHEERES, D.J. 1994. Dynamics about uniformly rotating tri-axial ellipsoids. Applications to asteroids. Icarus 110: 225-238.

16. Scheeres, D.J., S.J. Ostro, R.S. Hudson \& R.A. Werner. 1996. Orbits close to asteroid 4769 castalia. Icarus 121: 67-87. 
17. Scheeres, D.J., S.J. Ostro, R.S. Hudson, et al. 1998. Dynamics of orbits close to asteroid 4179 toutatis. Icarus 132: 53-79.

18. Scheeres, D.J., D.D. Durda \& P.E. Geissler. 2002. The fate of asteroid ejecta. In Asteroids III. W.M. Bottke, et al., Eds.: University of Arizona Press, Tuscon.

19. Scheeres, D.J., B.G. Williams \& J.K. Miller. 2000. Evaluation of the dynamic environment of an asteroid: applications to 433 eros. J. Guidance Cont. Dyn. 23: 466475.

20. Duboshin, G.N. 1958. The differential equations of translational-rotational motion of mutally attracting rigid bodies. Astronomicheskii Zhurnal 35: 265.

21. MaciejewsKi, A.J. 1995. Reduction, relative equilibria and potential in the two rigid bodies problem. Celest. Mech. Dyn. Astron. 63: 1-28.

22. ScheERES, D.J. 2002. Stability in the full two-body problem. Celest. Mech. Dyn. Astron. 83: 155-169.

23. Scheeres, D.J., S.J. Ostro, R.A. Werner, et al. 2000. Effects of gravitational interactions on asteroid spin states. Icarus 147: 106-118.

24. SCHEERES, D.J. 2001. Changes in rotational angular momentum due to gravitational interactions between two finite bodies. Celest. Mech. Dyn. Astron. 81: 39-44.

25. SCHEERES, D.J. 2002. Stability of binary asteroids. Icarus 159: 271-283.

26. BLoch, A.M. 2003. Nonhonomomic Mechanics and Control. Applied Mathematical Sciences Series. Springer-Verlag.

27. Marsden, J.E. \& T.S. Ratiu. 1999. Introduction to Mechanics and Symmetry. Texts in Applied Mathematics, 17: Springer-Verlag.

28. Marsden, J.E. 1992. Lectures on Mechanics, Vol. 174. London Math. Society, Lecture Note Series. Cambridge University Press.

29. Bloch, A.M., P.S. Krishnaprasad, J.E. Marsden \& T.S. Ratiu. 1994. Dissipation induced instabilities. Ann. Inst. H. Poincaré, Analyse Nonlineéaire 11: 37-90.

30. Bloch, A.M., P.S. Krishnaprasad, J.E. Marsden \& T.S. Ratiu. 1996. The EulerPoincaré equations and double bracket dissipation. Comm. Math. Phys. 175: 1-42.

31. Duncan, M., T. Quinn \& S. Tremaine. 1989. The long-term evolution of orbits in the solar system-a mapping approach. Icarus 82: 402-418.

32. Wisdom, J. \& M. Holman. 1991. Symplectic maps for the $n$-body problem. Astron. J. 102: $1528-1538$.

33. Lew, A., J.E. Marsden, M. Ortiz \& M. West. 2004. Variational time integration for mechanical systems. Intern. J. Num. Meth. Engin. To appear.

34. Lew, A., J.E. Marsden, M. Ortiz \& M. West. 2004. Asynchronous variational integrators. Arch. Rat. Mech. Anal. 167: 85-146.

35. JAFFÉ, C., S.D. Ross, M.W. Lo, et al. 2002. Statistical theory of asteroid escape rates. Phys. Rev. Lett. 89: 011101-1.

36. Dellnitz, M., O. Junge, W.-S. Koon, et al. 2003. Transport in dynamical astronomy and multibody problems. Preprint.

37. Néron de Surgy, O. \& J. Laskar. 1997. On the long term evolution of the spin of the earth. Astron. Astrophys. 318: 975-989.

38. Greenberg, R., G.V. Hoppa, P. Geissler, et al. 2002. The rotation of europa. Celest. Mech. Dyn. Astron. 83: 35-47.

39. Bottke, W.F., R. Jedicke, A. Morbidelli, et al. 2000. Understanding the distribution of near-Earth asteroids. Science 288: 2190-2194.

40. Bottke, W.F., D. Vokrouhlick , M. Brož, et al. 2001. Dynamical spreading of asteroid families by the yarkovsky effect. Science 294: 1693-1696.

41. Spitale, J. \& R. Greenberg. 2001. Numerical evaluation of the general yarkovsky effect: effects on semimajor axis. Icarus 149: 222-234.

42. RubinCAM, D. 2000. Radiative spin-up and spin-down of small asteroids. Icarus 148: $2-11$.

43. Neishtadt, A.I., D.J. Scheeres, V.V. Sidorenko \& A.A. Vasiliev. 2002. Evolution of comet nucleus rotation. Icarus 157: 205-218.

44. Yeomans, D.K. \& P.W. Chodas. 1989. An asymmetric outgassing model for cometary nongravitational accelerations. Astron. J. 98: 1083-1093.

45. Margot, J.L., M.C. Nolan, L.A.M. Benner, et al. 2002. Binary asteroids in the nearearth object population. Science 296: $1445-1448$. 
46. Veillet, C., J.W. Parker, I. Griffin, et al. 2002. The binary kuiper-belt object 1998 ww31. Nature 416: 711.

47. Merline, W.J., S.J. Weidenschilling, D.D. Durda, et al. 2002. Asteroids do have satellites. In Asteroids III. W.M. Bottke, A. Cellino, P. Paolicchi \& R.P. Binzel, Eds.: 289-312. University of Arizona Press, Tucson.

48. Belton, M.J.S., B.E.A. Mueller, L.A. D’Amario, et al. 1996. The discovery and orbit of 1993 (243)1 dactyl. Icarus 120: 185-199.

49. Benner, L.A.M., S.J. Ostro, J.D. Giorgini, et al. 2001. 1999 kw4. IAU Circular 7730.

50. HARRIS, A.W. 2002. On the slow rotation of asteroids. Icarus 156: 184-190.

51. SCHEERES, D.J. 1999. The effect of $c_{22}$ on orbit energy and angular momentum. Celest. Mech. Dyn. Astron. 73: 339-348.

52. Simo, J.C., D.R. Lewis \& J.E. MARSDEN. 1991. Stability of relative equilibria I: the reduced energy momentum method. Arch. Rat. Mech. Anal. 115: 15-59.

53. Marsden, J.E. \& M. West. 2001. Discrete mechanics and variational integrators. Acta Numerica 10: $357-514$

54. Koon, W.S., M.W. Lo, J.E. Marsden \& S.D. Ross. 2000. Heteroclinic connections between periodic orbits and resonance transitions in celestial mechanics. Chaos 10: 427-469.

55. Cendra, H., J.E. Marsden \& T.S. Ratiu. 2001. Lagrangian reduction by stages, volume 152 of Memoirs. American Mathematical Society, Providence.

56. Marsden, J.E., T.S. Ratiu \& J. Scheurle. 2000. Reduction theory and the lagrangerouth equations. J. Math. Phys. 41: 3379-3429.

57. Maciejewski, A.J. 1999. The two rigid bodies problem. reduction and relative equilibria. In Hamiltonian Systems with Three or More Degrees of Freedom (S'Agaró, 1995). 475-479.

58. GoŹDzIEwsKi, K. \& A.J. MACIEJEwsKi. 1999. Unrestricted planar prolbme of a symmetric body and a point mass. triangular libration points and their stability. Celest. Mech. Dyn. Astron. 75: 251-285.

59. LeOnARd, N.E. \& J.E. Marsden. 1997. Stability and drift of underwater vehicle dynamics: mechanical systems with rigid motion symmetry. Physica D 105: 130 162.

60. Marsden, J., G. Misiolek, M. Perlmutter \& T. Ratiu. 1998. Symplectic reduction for semidirect products and central extensions. Diff. Geom. Appl. 9: 173-212.

61. Marsden, J.E., R. Montgomery \& T.S. Ratiu. 1990. Reduction, symmetry and phases in mechanics. Memoirs of the AMS, Vol. 436. Amer. Math. Society, Providence.

62. Clerc, M. \& J.E. Marsden. 2001. Dissipation-induced instabilities in an optical cavity laser: a mechanical analog near the 1:1 resonance. Phys. Rev. E 64: 067603.

63. LARA, M. \& D.J. SCHEERES. 2002. Stability bounds for three-dimensional motion close to asteroids. J. Astronaut. Sci. 50(4): 389-409.

64. Alber, M.S., G.G. Luther, J.E. Marsden \& J.M. Robbins. 1998. Geometric phases, reduction and Lie-Poisson structure for the resonant three-wave interaction. Physica D 123: 271-290.

65. Hernandez, A. \& J.E. Marsden. 2004. Bifurcation of singular relative equilibria by the blowing up method. SIAM J. Appl. Dyn. Systems. To appear.

66. SteRn, S.A., W.F. BotTKE \& H.F. LEVISON. 2003. Regarding the putative eccentricity of charon's orbit. Astron. J. 125: 902-905.

67. Sawai, S., D.J. ScheERes \& S. Broschart. 2002. Control of hovering spacecraft using altimetry. J. Guidance Cont. Dyn. 25: 786-795.

68. Chang, D., A.M. Bloch, N. Leonard, et al. 2002. The equivalence of controlled lagrangian and controlled hamiltonian systems. Cont. Calc. Variations (special issue dedicated to J.L. Lions) 8: 393-422.

69. Rom-Kedar, V. \& S. Wiggins. 1990. Transport in two-dimensional maps. Arch. Rat. Mech. Anal. 109: 239-298.

70. Lekien, F. \& J.E. Marsden. 2004. Separatrices in high-dimensional phase spaces: application to van der waals dissociation. In preparation. 
71. Lekien, F. \& C. Coulliette. 2004. mangen: computation of hyperbolic trajectories, invariant manifolds and lobes of dynamical systems defined as $2 d+1$ data sets. In preparation.

72. Lekien, F. 2003. Time-Dependent Dynamical Systems and Geophysical Flows. Ph.D. Thesis, California Institute of Technology.

73. Wiggins, S. 1992. Chaotic Transport in Dynamical Systems. Interdisciplinary Appl. Math., Vol. 2. Springer, Berlin-Heidelberg-New York.

74. Rom-Kedar, V., A. LeOnard \& S. Wiggins. 1990. An analytical study of transport, mixing and chaos in an unsteady vortical flow. J. Fluid Mech. 214: 347-394.

75. Rom-Kedar, V. \& S. WigGins. 1991. Transport in two-dimensional maps: concepts, examples, and a comparison of the theory of rom-kedar and wiggins with the markov model of mackay, meiss, ott, and percival. Physica D 51: 248-266.

76. Coulliette, C. \& S. Wiggins. 2001. Intergyre transport in a wind-driven, quasigeostrophic double gyre: an application of lobe dynamics. Nonlinear Process. Geophys. 8: 69-94.

77. Lekien, F., C. Coulliette \& J.E. Marsden. 2004. Lagrangian structures in very high frequency radar data and optimal pollution timing. In Proc. 7th Experimental Chaos Conference. American Institute of Physics. 162-168.

78. TiYAPAN, A. \& C. JafFÉ. 1995. Chaotic scattering: an invariant fractal tiling of phase space. J. Chem. Phys. 103: 5499-5511.

79. Jalnapurkar, S.M., M. Leok, J.E. Marsden \& M. West. 2004. Discrete routh reduction. Found. Comput. Math. To appear.

80. Saha, P. \& S. Tremaine. 1992. Symplectic integrators for solar system dynamics. Astron. J. 104: 1633-1640.

81. Marsden, J.E., G.W. Patrick \& S. Shkoller. 1998. Multisymplectic geometry, variational integrators and nonlinear PDEs. Comm. Math. Phys. 199: 351-395.

82. Kane, C., J.E. Marsden, M. Ortiz \& M. West. 2000. Variational integrators and the newmark algorithm for conservative and dissipative mechanical systems. Int. J. Num. Meth. Eng. 49: 1295-1325.

83. Rowley, C.W. \& J.E. MARSDEN. 2002. Variational integrators for point vortices. Proc. CDC 40: 1521-1527.

84. SCHLICK, T. 2002. Molecular Modeling and Simulation. Interdisciplinary Applied Mathematics, Vol. 21. Springer-Verlag, New York.

85. Marsden, J.E., S. Pekarsky \& S. Shkoller. 2000. Symmetry reduction of discrete lagrangian mechanics on lie groups. J. Geom. Phys. 36: 140-151.

86. Dellnitz, M. \& O. Junge. 2002. Set oriented numerical methods for dynamical systems. In Handbook of Dynamical Systems, Vol. 2. 221-264. North-Holland, Amsterdam.

87. Murray, C.D. \& S.F. DermotT. 1999. Solar System Dynamics. Cambridge.

88. Cho, S.N., H. McClamroch \& M. Reyhanoglu. 2000. Dynamics of multi-body vehicles and their formulation as nonlinear control systems. In Proc. 2000 American Control Conference. 3908-3912.

89. Hagerty, P., A.M. Bloch \& M. Weinstein. 1999. Radiation induced instability in interconnected systems. In Proc. 38th Conf. Decisions Control. IEEE 651-656.

90. Washabaugh, P.D. \& D.J. Scheeres. 2002. Energy and stress distributions in ellipsoids. Icarus 159: 314-321.

91. Asphaug, E. \& W. BenZ. 1996. Size, density, and structure of comet shoemaker-levy 9 inferred from the physics of tidal breakup. Icarus 121: 225-248.

92. Richardson, D.C., W.F. BotTKe \& S.G. Love. 1998. Tidal distortion and disruption of Earth-crossing asteroids. Icarus 134: 47-76.

93. HARRIS, A.W. 1994. Tumbling asteroids. Icarus 107: 209-211.

94. Bottke, W.F. \& H.J. Melosh. 1996. The formation of binary asteroids and doublet craters. Icarus 124: 372-391.

95. DuRDA, D.D. 1996. The formation of asteroidal satellites in catastrophic collisions. Icarus 120: 212-219.

96. Doressoundiram, A., P. Paolicchi, A. Verlicchi \& A. Cellino. 1997. The formation of binary asteroids as outcomes of catastrophic collisions. Planet. Space. Sci. 45: $757-770$. 
97. BottKe, W.F., D.C. Richardson, P. Michel \& S.G. Love. 1999. 1620 geographos and 433 eros: shaped by planetary tides? Astron. J. 117: 1921-1928.

98. Michel, P., W. Benz, P. TAnga \& D.C. Richardson. 2001. Collisions and gravitational reaccumulation: forming asteroid families and satellites. Science 294: 16961700 .

99. Goldreich, P., Y. Lithwick \& R. Sari. 2002. Formation of kuiper-belt binaries by dynamical friction and three-body encounters. Nature 240: 643-646.

100. Chandrasekhar, S. 1969. Ellipsoidal Figures of Equilibrium. Yale University Press.

101. Bloch, A.M., D. Chang, N. LeOnARD \& J.E. MARSDEN. 2001. Controlled lagrangians and the stabilization of mechanical systems ii: potential shaping. IEEE Trans. Auto. Cont. 46: $1556-1571$.

102. Shen, J., N.H. McClamroch \& A.M. Bloch. 2004. Local equilibrium controllability of multibody systems controlled via shape change. IEEE Trans. Auto. Cont. To appear.

103. MeISS, J.D. 1992. Symplectic maps, variational principles, and transport. Rev. Mod. Phys. 64: 795-848.

104. Rom-Kedar, V. 1999. Transport in a class of $n$-d.o.f. systems. In Hamiltonian Systems with Three or More Degrees of Freedom (S'Agaró, 1995). 538-543.

105. Beigie, D., A. Leonard \& S. Wiggins. 1991. A global study of enhanced stretching and diffusion in chaotic tangles. Phys. Fluids A 3: 1039-1049.

106. Malhotra, N. \& S. Wiggins. 1998. Geometric structures, lobe dynamics, and lagrangian transport in flows with aperiodic time dependence, with applications to rossby wave flow. J. Nonlinear Sci. 8: 401-456.

107. Poje, A.C. \& G. Haller. 1999. Geometry of cross-stream mixing in a double-gyre ocean model. Phys. Oceanogr. 29: 1649-1665.

108. Haller, G. \& G. YuAN. 2000. Lagrangian coherent structures and mixing in twodimensional turbulence. Physica D 147: 352-370.

109. Haller, G. 2001. Distinguished material surfaces and coherent structures in $3 \mathrm{~d}$ fluid flows. Physica D 149: 248-277.

110. JafFé, C., D. FARRELly \& T. UZER. 2000. Transition state theory without time-reversal symmetry: chaotic ionization of the hydrogen atom. Phys. Rev. Lett. 84: 610-613.

111. Ross, S.D., W.S. Koon, M.W. Lo \& J.E. MArsden. 2003. Design of a multi-moon orbiter. In 13th AAS/AIAA Space Flight Mechanics Meeting, Ponce, Puerto Rico, AAS 03-143. 